\title{
First-principles approach to noncollinear magnetism: Towards spin dynamics
}

Sharma, S.; Dewhurst, J.K.; Ambrosch-Draxl, C.; Kurth, S.; Helbig, N.; Pittalis, S.; Shallcross, Sam; Nordstrom, L.; Gross, E.K.U.

Published in:

Physical Review Letters

Link to article, DOI:

10.1103/PhysRevLett.98.196405

Publication date:

2007

Document Version

Publisher's PDF, also known as Version of record

Link back to DTU Orbit

Citation (APA):

Sharma, S., Dewhurst, J. K., Ambrosch-Draxl, C., Kurth, S., Helbig, N., Pittalis, S., Shallcross, S., Nordstrom, L., \& Gross, E. K. U. (2007). First-principles approach to noncollinear magnetism: Towards spin dynamics. Physical Review Letters, 98(19), 196405. https://doi.org/10.1103/PhysRevLett.98.196405

\section{General rights}

Copyright and moral rights for the publications made accessible in the public portal are retained by the authors and/or other copyright owners and it is a condition of accessing publications that users recognise and abide by the legal requirements associated with these rights.

- Users may download and print one copy of any publication from the public portal for the purpose of private study or research.

- You may not further distribute the material or use it for any profit-making activity or commercial gain

- You may freely distribute the URL identifying the publication in the public portal 


\title{
First-Principles Approach to Noncollinear Magnetism: Towards Spin Dynamics
}

\author{
S. Sharma, ${ }^{1,2,5, *}$ J. K. Dewhurst, ${ }^{2,3}$ C. Ambrosch-Draxl, ${ }^{2,4}$ S. Kurth, ${ }^{5}$ N. Helbig, ${ }^{1,5}$ S. Pittalis, ${ }^{5}$ S. Shallcross, ${ }^{6}$ \\ L. Nordström, ${ }^{7}$ and E. K. U. Gross ${ }^{5}$ \\ ${ }^{1}$ Fritz Haber Institute of the Max Planck Society, Faradayweg 4-6, D-14195 Berlin, Germany \\ ${ }^{2}$ Institut für Physik, Karl-Franzens-Universität Graz, Universitätsplatz 5, A-8010 Graz, Austria \\ ${ }^{3}$ School of Chemistry, The University of Edinburgh, Edinburgh EH9 3JJ, United Kingdom \\ ${ }^{4}$ Department Materials Physics, University of Leoben, Erzherzog Johann-Strasse 3, A-8700 Leoben, Austria \\ ${ }^{5}$ Institut für Theoretische Physik, Freie Universität Berlin, Arnimallee 14, D-14195 Berlin, Germany \\ ${ }^{6}$ Department of Physics, Technical University of Denmark, DK-2800 Lyngby, Denmark \\ ${ }^{7}$ Department of Physics, Uppsala University, Box 530, 75121 Uppsala, Sweden
}

(Received 29 August 2006; published 9 May 2007)

\begin{abstract}
A description of noncollinear magnetism in the framework of spin-density functional theory is presented for the exact exchange energy functional which depends explicitly on two-component spinor orbitals. The equations for the effective Kohn-Sham scalar potential and magnetic field are derived within the optimized effective potential (OEP) framework. With the example of a magnetically frustrated $\mathrm{Cr}$ monolayer it is shown that the resulting magnetization density exhibits much more noncollinear structure than standard calculations. Furthermore, a time-dependent generalization of the noncollinear OEP method is well suited for an ab initio description of spin dynamics. We also show that the magnetic moments of solids $\mathrm{Fe}, \mathrm{Co}$, and $\mathrm{Ni}$ are well reproduced.
\end{abstract}

DOI: 10.1103/PhysRevLett.98.196405

PACS numbers: 71.15.Mb, 71.10.- w, 71.22.+i

The extension of the original density functional theory (DFT) Hohenberg-Kohn-Sham approach to the case of spin polarized systems was given under the name spin DFT (SDFT) more than three decades ago [1]. While this formulation was for arbitrary directions of the magnetization vector field, even today most applications are based on a restricted collinear version. This has the advantage of computational simplicity: one then works with two separate Kohn-Sham (KS) equations, one yielding the spin-up orbitals the other the spin-down orbitals, whereas the general formulation involves Pauli spinors. Nevertheless, there exists a wealth of noncollinearity in nature. To give only a few examples, it is widely seen in molecular magnets, exchange frustrated solids ( $\gamma$-Fe, spin glasses), and all magnets at finite temperatures.

Crucial for practical calculations using SDFT is the approximation made for the exchange-correlation (XC) energy functional. The Local Spin-Density Approximation (LSDA) and the Generalized Gradient Approximations (GGAs) are currently the most popular ones. These have been developed for collinear magnetism, and their use in noncollinear situations relies on the magnetization, $\mathbf{m}(\mathbf{r})$, and exchange-correlation magnetic field, $\mathbf{B}_{\mathrm{XC}}(\mathbf{r})$, being made collinear in a local reference frame at each point in space [2]. This is only possible with purely local functionals like LSDA [3,4], though it has been used under additional approximations for gradient functionals as well [5]. Such approximations (that lead to locally collinear magnetization and $\mathrm{XC}$ magnetic field) cause $\mathbf{m}(\mathbf{r}) \times$ $\mathbf{B}_{\mathrm{XC}}(\mathbf{r})$ to vanish everywhere in space. As noted recently, this fact renders the adiabatic time-dependent extension of these functionals improper [6] for the study of spin dy- namics because in the absence of external magnetic fields and within adiabatic approximation, the local torque on the spins $\left[\mathbf{m}(\mathbf{r}, \mathbf{t}) \times \mathbf{B}_{\mathrm{XC}}(\mathbf{r}, \mathbf{t})\right]$ vanishes [7]. This is a serious limitation since the dynamics of the spin degree of freedom is responsible for a number of important phenomena such as spin injection, the dynamics of Bloch walls, spin wave excitations [8], and spin filtering, mechanisms crucial for recent developments in spintronics [9]. The search for approximate XC functionals which depend on all three components of the spin magnetization $\mathbf{m}$ beyond the form of the locally collinear LSDA has remained a major challenge in the description of noncollinear magnetism.

In recent years, an alternative route to the construction of approximate $\mathrm{XC}$ functionals has enjoyed increasing interest. These involve functionals depending explicitly on the single-particle KS orbitals which, through the KS singleparticle equation, are implicit functionals of the density [10]. Technically, one needs to employ the Optimized Effective Potential (OEP) [11] method to compute the local $\mathrm{XC}$ potential. The simplest orbital-dependent approximation to the XC energy is the EXact eXchange (EXX) functional which is the Fock exchange energy but evaluated with KS orbitals (i.e., orbitals coming from a local potential). A number of successful EXX calculations have been reported for semiconductors [12-14] and magnetic metals [15]. However, for magnetic systems, again the collinear formalism has been employed.

In this Letter, we extend the OEP formalism for SDFT to noncollinear magnetic systems. Most importantly, we do not rely on a condition of local collinearity and treat the wave functions as Pauli spinors for high lying and Dirac spinors for deep lying (3 Ha below the Fermi level) elec- 
trons. Using the EXX functional, we demonstrate with the example of an unsupported $\mathrm{Cr}(111)$ monolayer, that (i) the magnetization and $\mathbf{B}_{\mathrm{XC}}$ are generally not locally parallel in contrast to what has been assumed in all calculations to date, and (ii) that the noncollinearity is much more pronounced than found with the LSDA functional. Against popular belief [16], we find that this noncollinearity is not restricted to just the interstitial region but spreads all the way to the atom center. With the examples of bulk Fe, Co, and $\mathrm{Ni}$, we further show that our formalism can also be effectively used for collinear magnets.

To derive the OEP equations in the general noncollinear case, we start with the KS equation for two-component spinors $\Phi_{i}$, which has the form of a Pauli equation. For noninteracting electrons moving in an effective scalar potential $v_{\mathrm{s}}$ and a magnetic vector field $\mathbf{B}_{\mathbf{s}}$, it reads as (atomic units are used throughout)

$$
\left(-\frac{1}{2} \nabla^{2}+v_{\mathrm{s}}(\mathbf{r})+\mu_{B} \boldsymbol{\sigma} \cdot \mathbf{B}_{\mathrm{s}}(\mathbf{r})\right) \Phi_{i}(\mathbf{r})=\varepsilon_{i} \Phi_{i}(\mathbf{r}) .
$$

This equation can be derived by minimizing the total energy which, in SDFT, is given as a functional of the density $\rho(\mathbf{r})=\sum_{i}^{\text {occ }} \Phi_{i}^{\dagger}(\mathbf{r}) \Phi_{i}(\mathbf{r})$ and the magnetization density $\mathbf{m}(\mathbf{r})=\mu_{B} \sum_{i}^{\text {occ }} \Phi_{i}^{\dagger}(\mathbf{r}) \boldsymbol{\sigma} \Phi_{i}(\mathbf{r})$. For a given external scalar potential $v_{\text {ext }}$ and magnetic field $\mathbf{B}_{\text {ext }}$, this total energy reads

$$
\begin{aligned}
E[\rho, \mathbf{m}]= & T_{s}[\rho, \mathbf{m}]+\int \rho(\mathbf{r}) v_{\mathrm{ext}}(\mathbf{r}) d \mathbf{r} \\
& +\int \mathbf{m}(\mathbf{r}) \cdot \mathbf{B}_{\mathrm{ext}}(\mathbf{r}) d \mathbf{r}+U[\rho]+E_{\mathrm{XC}}[\rho, \mathbf{m}] \\
= & \sum_{i}^{\mathrm{occ}} \varepsilon_{i}-\int \rho(\mathbf{r}) v_{\mathrm{XC}}(\mathbf{r}) d \mathbf{r} \\
& -\int \mathbf{m}(\mathbf{r}) \cdot \mathbf{B}_{\mathrm{XC}}(\mathbf{r}) d \mathbf{r}-U[\rho]+E_{\mathrm{XC}}[\rho, \mathbf{m}],
\end{aligned}
$$

where $U[\rho]=1 / 2 \int \rho(\mathbf{r}) \rho\left(\mathbf{r}^{\prime}\right) /\left|\mathbf{r}-\mathbf{r}^{\prime}\right| d \mathbf{r} d \mathbf{r}^{\prime} \quad$ is the Hartree energy. The XC potential and XC magnetic field are given by

$$
v_{\mathrm{XC}}(\mathbf{r})=\frac{\delta E_{\mathrm{XC}}[\rho, \mathbf{m}]}{\delta \rho(\mathbf{r})} \quad \text { and } \quad \mathbf{B}_{\mathrm{XC}}(\mathbf{r})=\frac{\delta E_{\mathrm{XC}}[\rho, \mathbf{m}]}{\delta \mathbf{m}(\mathbf{r})},
$$

respectively. The exact functional form of $E_{\mathrm{XC}}[\rho, \mathbf{m}]$ is unknown and has to be approximated in practice.

Assuming that the densities $(\rho, \mathbf{m})$ are noninteracting $(v, \mathbf{B})$-representable one may, equivalently, minimize the total-energy functional (2) over the effective scalar potential and magnetic field. Thus, the conditions

$$
\left.\frac{\delta E[\rho, \mathbf{m}]}{\delta v_{\mathrm{s}}(\mathbf{r})}\right|_{\mathbf{B}_{\mathrm{s}}}=0 \quad \text { and }\left.\quad \frac{\delta E[\rho, \mathbf{m}]}{\delta \mathbf{B}_{\mathrm{s}}()}\right|_{v_{\mathrm{s}}}=0
$$

must be satisfied.
If the functional derivatives in Eq. (4) are evaluated for an XC functional that depends explicitly on the KS spinors, one obtains the natural extension of the OEP equations to noncollinear magnetism. By the usage of spinor valued wave functions, we can stay within a single global reference frame, in contrast to the case where functionals originally designed for collinear magnetism are used in a noncollinear context by introducing a local reference frame at each point in space. The most commonly used orbital functional is the EXX energy given by

$$
E_{\mathrm{x}}^{\operatorname{EXX}}\left[\left\{\Phi_{i}\right\}\right] \equiv-\frac{1}{2} \iint \sum_{i, j}^{\mathrm{occ}} \frac{\Phi_{i}^{\dagger}(\mathbf{r}) \Phi_{j}(\mathbf{r}) \Phi_{j}^{\dagger}\left(\mathbf{r}^{\prime}\right) \Phi_{i}\left(\mathbf{r}^{\prime}\right)}{\left|\mathbf{r}-\mathbf{r}^{\prime}\right|} d \mathbf{r} d \mathbf{r}^{\prime}
$$

where the label occ indicates that the summation runs only over occupied states. In the following, we restrict ourselves to an exchange-only treatment although generalization to other orbital functionals is straightforward.

For the energy functional Eq. (2) using the EXX approximation to $E_{\mathrm{XC}}$, one obtains the following coupled integral equations for the exchange potential and magnetic field:

$$
\left.R_{v}(\mathbf{r}) \equiv \frac{\delta E[\rho, \mathbf{m}]}{\delta v_{\mathrm{s}}(\mathbf{r})}\right|_{\mathbf{B}_{\mathrm{s}}}=\sum_{i}^{\mathrm{occ}} \sum_{j}^{\text {un }}\left(\Lambda_{i j} \frac{\rho_{i j}(\mathbf{r})}{\varepsilon_{i}-\varepsilon_{j}}+\text { c.c. }\right)=0
$$

and

$$
\left.\mathbf{R}_{\mathbf{B}}(\mathbf{r}) \equiv \frac{\delta E[\rho, \mathbf{m}]}{\delta \mathbf{B}_{\mathrm{s}}(\mathbf{r})}\right|_{v_{\mathrm{s}}}=\sum_{i}^{\text {occ }} \sum_{j}^{\text {un }}\left(\Lambda_{i j} \frac{\mathbf{m}_{i j}(\mathbf{r})}{\varepsilon_{i}-\varepsilon_{j}}+\text { c.c. }\right)=0,
$$

where $\rho_{i j}(\mathbf{r})=\Phi_{i}^{\dagger}(\mathbf{r}) \Phi_{j}(\mathbf{r}), \quad \mathbf{m}_{i j}(\mathbf{r})=\mu_{B} \Phi_{i}^{\dagger}(\mathbf{r}) \boldsymbol{\sigma} \Phi_{j}(\mathbf{r})$, and $j$ runs only over the unoccupied states. The matrix $\Lambda$ is given by

$$
\Lambda_{i j}=\left(V_{i j}^{\mathrm{NL}}\right)^{\dagger}-\int \rho_{i j}^{\dagger}(\mathbf{r}) v_{\mathrm{x}}(\mathbf{r}) d \mathbf{r}-\int \mathbf{m}_{i j}^{\dagger}(\mathbf{r}) \cdot \mathbf{B}_{\mathrm{x}}(\mathbf{r}) d \mathbf{r},
$$

where

$$
V_{i j}^{\mathrm{NL}}=-\sum_{k}^{\mathrm{occ}} \iint \frac{\Phi_{i}^{\dagger}(\mathbf{r}) \Phi_{k}(\mathbf{r}) \Phi_{k}^{\dagger}\left(\mathbf{r}^{\prime}\right) \Phi_{j}\left(\mathbf{r}^{\prime}\right)}{\left|\mathbf{r}-\mathbf{r}^{\prime}\right|} d \mathbf{r} d \mathbf{r}^{\prime},
$$

are the nonlocal matrix elements of the Coulomb interaction between states $i$ and $j$.

To ensure that our numerical analysis be as accurate as possible, we use the full-potential linearized augmented plane wave (FP-LAPW) method [17] implemented within the EXCITING code [18]. Here, the single electron potential is calculated exactly without any shape approximation, and the space is divided into muffin-tin (MT) regions, where atomic orbitals are used as a basis and interstitial region, where plane waves are used as a basis. The deep lying core 
states (3 Ha below the Fermi level) are treated as Dirac spinors and valence states as Pauli spinors. More importantly, the magnetization density and XC magnetic field are both treated as unconstrained vector fields throughout space. In our implementation of the OEP method, the exchange fields are iteratively updated by subtracting the residue functions $R_{v}$ and $\mathbf{R}_{\mathbf{B}}$ from the exchange fields. In other words, if $i$ is the iteration number, then

$$
v_{\mathrm{x}}^{i}(\mathbf{r})=v_{\mathrm{x}}^{i-1}(\mathbf{r})-\tau R_{v}^{i}(\mathbf{r}), \mathbf{B}_{\mathrm{x}}^{i}(\mathbf{r})=\mathbf{B}_{\mathrm{x}}^{i-1}(\mathbf{r})-\tau \mathbf{R}_{\mathbf{B}}^{i}(\mathbf{r})
$$

is repeated until convergence is reached, with $R_{v}^{i}$ and $\mathbf{R}_{\mathbf{B}}^{i}$ calculated by inserting $v_{\mathrm{x}}^{i-1}$ and $\mathbf{B}_{\mathrm{x}}^{i-1}$ into Eqs. (6) and (7). $\tau$ is the mixing chosen in such a manner as to achieve a speedy convergence. In the collinear case, this method is similar to the one previously suggested in Ref. [19].

In order to explore the impact of treating noncollinear magnetism in the way outlined above, we compare our approach with the standard LSDA functional using the example of an unsupported $\mathrm{Cr}$ (111) monolayer. We set the lattice parameter of the $\mathrm{Cr}$ monolayer to that of the $\mathrm{Ag}$ (111) surface. The result is a topologically frustrated antiferromagnet, known from LSDA calculations to exist as a noncollinear Néel state with the net magnetization direction of the three nonequivalent atoms pointing at $120^{\circ}$ to each other. In Fig. 1, we show the magnetization density and $\mathbf{B}$ field for both the LSDA and EXX functionals. Both find, as they must, the noncollinear Néel state, and in fact the EXX and LSDA MT averaged moments are similar, being $2.60 \mu_{B}$ and $2.0 \mu_{B}$, respectively. The details of the $\mathrm{XC}$ density and field however are very different with the EXX functional producing a lot more structure, in contrast to its fairly homogeneous LSDA counterpart. In the past, the LSDA results (of the kind shown in Fig. 1), which show
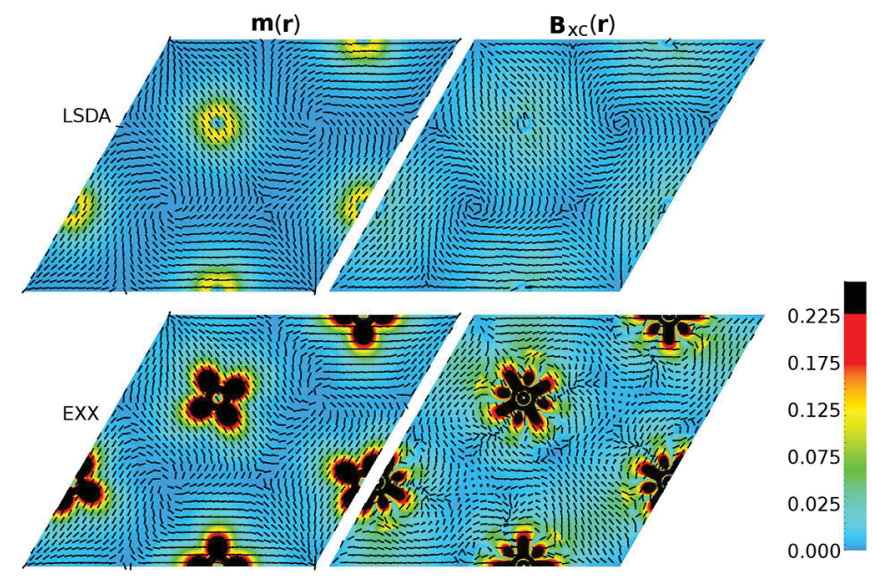

FIG. 1 (color). Fully noncollinear magnetization density and B field obtained using the LSDA and exchange-only EXX functionals for an unsupported $\mathrm{Cr}$ monolayer in Néel state. Arrows indicate the direction, and information about the magnitude (in atomic units) is given in the color bar. almost no noncollinearity in the MT region, led to the conclusion that it is sufficient to treat only the interstitial region as noncollinear [16]. The present Letter shows that orbital functionals such as EXX are more sensitive to the atomic shell structure, and this sensitivity also manifests itself in the magnetization density and exchange $\mathbf{B}$ field. This is clear from the flower petal-like structure visible in the magnitude of EXX density and $\mathbf{B}$ field. The Néel walls are also much narrower in the EXX case. Adding LSDA correlations to the EXX functional does not significantly change these results. A striking feature of the EXX $\mathbf{B}$ field is that, unlike its LSDA counterpart, it is not locally parallel to the magnetization density.

Another appealing property of the EXX functional that could have consequences in future time-dependent extensions is the nonvanishing cross product of the magnetization density and EXX $\mathbf{B}_{x}$ field. This is interesting because the equation of motion for the spin magnetization reads

$$
\frac{d \mathbf{m}(\mathbf{r}, t)}{d t}=\gamma \mathbf{m}(\mathbf{r}, t) \times\left[\mathbf{B}_{\mathrm{XC}}(\mathbf{r}, t)+\mathbf{B}_{\mathrm{ext}}(\mathbf{r}, t)\right]-\nabla \cdot \mathbf{J}_{s}
$$

where $\mathbf{J}_{s}$ is the spin current and $\gamma$ the gyromagetic ratio. In the time-independent LSDA and conventional GGA, $\mathbf{m}(\mathbf{r})$ and $\mathbf{B}_{\mathrm{XC}}(\mathbf{r})$ are locally collinear, as is clear from Fig. 1, and therefore $\mathbf{m}(\mathbf{r}) \times \mathbf{B}_{\mathrm{XC}}(\mathbf{r})$ vanishes. This also holds true in the adiabatic approximation of time-dependent SDFT which, by Eq. (11), implies that these functionals cannot properly describe the dynamics of the spin magnetization. In contrast, already at the static level, for the EXX functional $\mathbf{m}(\mathbf{r}) \times \mathbf{B}_{\mathrm{x}}(\mathbf{r})$ does not vanish (see Fig. 2). In fact, in the ground state of a noncollinear ferromagnet without external magnetic field, $\mathbf{m}(\mathbf{r}) \times \mathbf{B}_{\mathrm{XC}}(\mathbf{r})$ exactly cancels the divergence of the spin current, $\nabla \cdot \mathbf{J}_{s}$; i.e., these terms are equally important, and it is essential to have a proper

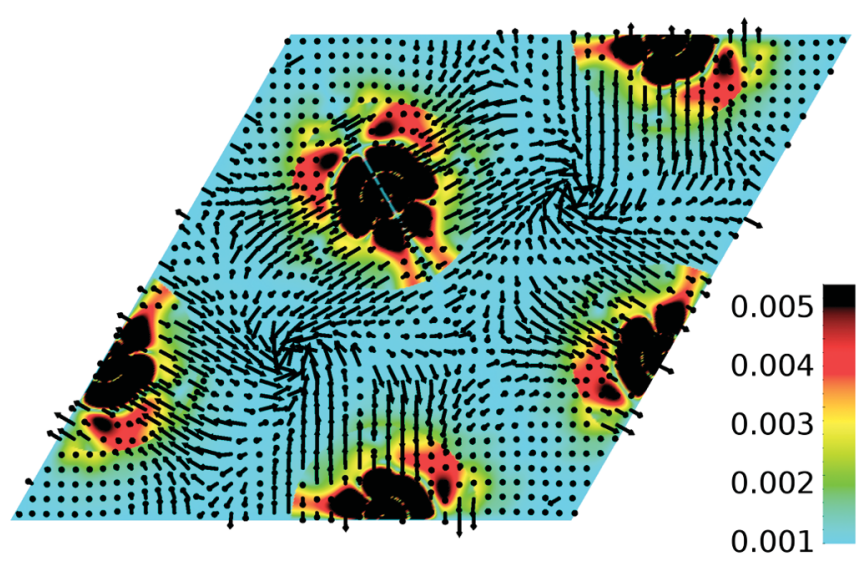

FIG. 2 (color). $\quad \mathbf{m}(\mathbf{r}) \times \mathbf{B}_{\mathrm{x}}(\mathbf{r})$ for an unsupported $\mathrm{Cr}$ monolayer, in the same plane as Fig. 1, obtained using the EXX functional. Arrows indicate the direction, and information about the magnitude (in atomic units) is given in the color bar. 
description of $\mathbf{m}(\mathbf{r}) \times \mathbf{B}_{\mathrm{XC}}(\mathbf{r})$. These results indicate that a time-dependent generalization of our method could open the way to an ab initio description of spin dynamics. How well this functional really performs in describing the spin dynamics remains a question for future investigations.

We now turn to the question of the calculation of magnetic moments of collinear solids with the present formalism using the EXX functional. For the collinear magnets $\mathrm{Fe}, \mathrm{Co}$, and $\mathrm{Ni}$, we find moments of $2.71 \mu_{B}\left(2.12 \mu_{B}\right)$, $1.77 \mu_{B}\left(1.71 \mu_{B}\right)$, and $0.50 \mu_{B}\left(0.55 \mu_{B}\right)$, respectively, where the LSDA results are indicated in brackets. Surprisingly, a previous OEP calculations $[15,20]$ found much larger moments of $3.40 \mu_{B}, 2.25 \mu_{B}$, and $0.68 \mu_{B}$, respectively. This discrepancy may be attributed to the following facts: first, the previous calculations used the atomic sphere approximation for the scalar potential and the atomic moment approximation for the magnetization. In our Letter, there is no shape approximation for the scalar potential, and the magnetization is treated as an unconstrained vector field. Second, and more important, in the present Letter, a coupled set of equations is solved to numerically invert the response function. This has the advantage of automatically including the response of the system to a constant magnetic field which is important for spin-unsaturated systems. This response needs additional treatment in the case where a decoupled set of equations is used and the response is inverted in a constant-free basis, as done in all past calculations $[15,20]$. We suspect that this is the major reason for the present discrepancy.

To conclude, we have presented a generalization of the widely used OEP equations for noncollinear magnetic systems. The resulting method does not need any assumption of local collinearity for $\mathbf{m}(\mathbf{r})$ and $\mathbf{B}_{\mathrm{XC}}(\mathbf{r})$, and therefore extends $a b$ initio approaches to noncollinear magnetism substantially beyond the LSDA. In particular, a time-dependent extension of the noncollinear OEP method naturally leads to a new and promising ab initio approach to describe spin dynamics.

Finally, we note that since the formalism presented here treats KS wave functions as spinors, it can be used in conjunction with spin-orbit coupling. In particular, in $f$-electron systems, both spin-orbit coupling and the exchange field are of crucial importance, where the latter is well known to be poorly treated by LSDA/GGA. Hence, the present Letter opens new interesting routes for future extensions.
We acknowledge the Austrian Science Fund (Project No. P16227), the EXCITING network funded by the EU (Contract No. HPRN-CT-2002-00317), NoE NANOQUANTA Network (Contract No. NMP4-CT2004-50019), Deutsche Forschungsgemeinschaft, and Swedish Research Council for financial support.

*Electronic address: sangeeta.sharma@physik.fu-berlin.de

[1] U. von Barth and L. Hedin, J. Phys. C 5, 1629 (1972).

[2] J. Kübler, K.-H. Höck, J. Sticht, and A. R. Williams, J. Phys. F 18, 469 (1988).

[3] L. Nordström and D. J. Singh, Phys. Rev. Lett. 76, 4420 (1996).

[4] T. Oda, A. Pasquarello, and R. Car, Phys. Rev. Lett. 80, 3622 (1998).

[5] E. Sjöstedt and L. Nordström, Phys. Rev. B 66, 014447 (2002).

[6] K. Capelle, G. Vignale, and B. L. Györffy, Phys. Rev. Lett. 87, 206403 (2001).

[7] K. Capelle and B. L. Györffy, Europhys. Lett. 61, 354 (2003).

[8] R. Gebauer and S. Baroni, Phys. Rev. B 61, R6459 (2000); N. M. Rosengaard and B. Johansson, Phys. Rev. B 55, 14975 (1997); S. V. Halilov, A. Y. Perlov, P. M. Oppeneer, and H. Eschrig, Europhys. Lett. 39, 91 (1997); S. Y. Savrasov, Phys. Rev. Lett. 81, 2570 (1998).

[9] S. A. Wolf et al., Science 294, 1488 (2001).

[10] M. Grüning, A. Marini, and A. Rubio, J. Chem. Phys. 124, 154108 (2006).

[11] J.D. Talman and W.F. Shadwick, Phys. Rev. A 14, 36 (1976).

[12] M. Städele, M. Moukara, J. A. Majewski, P. Vogl, and A. Görling, Phys. Rev. B 59, 10031 (1999).

[13] R. J. Magyar, A. Fleszar, and E. K. U. Gross, Phys. Rev. B 69, 045111 (2004).

[14] S. Sharma, J. K. Dewhurst, and C. Ambrosch-Draxl, Phys. Rev. Lett. 95, 136402 (2005).

[15] T. Kotani, J. Phys. Condens. Matter 10, 9241 (1998).

[16] P. Kurz, F. Förster, L. Nordström, G. Bihlmayer, and S. Blügel, Phys. Rev. B 69, 024415 (2004).

[17] D. J. Singh, Planewaves Pseudopotentials and the LAPW Method (Kluwer Academic Publishers, Boston, 1994).

[18] J. K. Dewhurst, S. Sharma, and C. Ambrosch-Draxl, URL http://exciting.sourceforge.net.

[19] S. Kümmel and J.P. Perdew, Phys. Rev. B 68, 035103 (2003).

[20] T. Kotani and H. Akai, Physica B (Amsterdam) 237, 332 (1997). 